\title{
Properties of the Ideal Ginzburg-Landau Vortex Lattice
}

\author{
Ernst Helmut Brandt \\ Max-Planck-Institut für Metallforschung, D-70506 Stuttgart, Germany
}

(Dated: October 30, 2018)

\begin{abstract}
The magnetization curves $M(H)$ for ideal type-II superconductors and the maximum, minimum, and saddle point magnetic fields of the vortex lattice are calculated from Ginzburg-Landau theory for the entire ranges of applied magnetic fields $H_{c 1} \leq H \leq H_{c 2}$ or induction $0 \leq B \leq \mu_{0} H_{c 2}$ and Ginzburg-Landau parameters $2^{-1 / 2} \leq \kappa \leq 1000$. Results for the triangular and square flux-line lattices are compared with the results of the circular cell approximation. The exact magnetic field $B(x, y)$ and magnetization $M(H, \kappa)$ are compared with often used approximate expressions, some of which deviate considerably or have limited validity. Useful limiting expressions and analytical interpolation formulas are presented.
\end{abstract}

PACS numbers: 74.25.Qt, 74.25.Ha, 74.20.De

\section{INTRODUCTION}

Since Abrikosov's 1 prediction of the flux-line lattice in Type-II superconductors from Ginzburg-Landau (GL) theor $\mathrm{y}^{2}$, several approximate formulas for the magnetization $M=B / \mu_{0}-H$ versus the applied magnetic field $H$ or average induction $B$ have been published $1,3,4,5,6,7$. In these papers and below, the basic situation is considered where a macroscopically large, homogeneous and isotropic, long superconductor is exposed to a uniform parallel field $H$. In this ideal case demagnetization effects, flux-line pinning, and surface effects may be disregarded, and thus the flux-lines are straight lines forming an ideal periodic lattice. These results are easily extended to anisotropic superconductors (where an anisotropic effective-mass tensor is introduced into the GL theory) by defining an effective GL parameter $\tilde{\kappa}$ that depends on the orientation of the flux lines; this transformation works when $H$ is along a principle symmetry axis $8,9,10$. Generalization to geometries where demagnetization effects occur, are possible by introduction of a demagnetizing factor; but this concept works only for homogeneous specimens with the shape of an ellipsoid. In this case the flux lines in the bulk are still straight and form an ideal flux-line lattice (FLL). For other specimen shapes the FLL is distorted, i.e., the orientation and density of the FLL varies spatially and can be calculated only numerically 11.12 .

The aim of the present paper is to compare the widely used approximate expressions for $M(H, \kappa)$ with the exact value obtained numerically and to give useful general analytic interpolation formulas valid in the entire ranges of $H$ and $\kappa$ where the FLL exists, namely, $H_{c 1} \leq H \leq H_{c 2}$ for $H$, or $0 \leq B \leq B_{c 2}=\mu_{0} H_{c 2}$, and $1 / \sqrt{2} \leq \kappa<\infty$ for $\kappa$, where $H_{c 1}(T)$ and $H_{c 2}(T)$ are the lower and upper critical fields and $\kappa$ is the GL parameter. Interestingly, such general formulas have not been published yet, and thus the accuracy of the commonly used expressions is not known, probably due to the difficulty of the numerical solution of the complex-valued GL equations. Early numerics ${ }^{13}$ used the circular cell method (CCM), which approximates the hexagonal unit cell of the triangular
FLL (or the quadratic unit cell of the square FLL) by a circle and the two-dimensional (2D) solution by the 1D rotationally symmetric solution inside this circular cell; both the GL function and the magnetic field are forced to have vanishing slope on this circular boundary, as the exact solution has on the boundary of the Wigner-Seitz cell. This method yields the exact $H_{c 1}$ and is expected to be best at low inductions $B \ll B_{c 2}$ where the flux lines are well separated. But surprisingly, the circular cell approximation gives very good magnetization curves at all $B$ (see Fig. 1) and even yields the exact value of the upper critical field $H_{c 2}$. Some more exact results of the CCM are listed below. Another $\operatorname{method}^{7}$ uses a similar circular symmetric GL order parameter and a linear superposition of circular symmetric magnetic fields to obtain excellent approximate $M(H, \kappa)$, see also Ref. 14 .

An in principle exact numerical method ${ }^{15}$ uses periodic real trial functions for the squared GL function $|\psi(x, y)|^{2}$ and magnetic field $B(x, y)$ and minimizes the resulting free energy functional with respect to a finite number of Fourier coefficients. The same method was later applied ${ }^{16}$ to solve the microscopic BCS-Gor'kov theory for the properties of the FLL in the entire temperature interval $0 \leq T \leq T_{c}$ where $T_{c}$ is the superconducting transition temperature (GL theory, strictly spoken, applies only close to $T_{c}$ ). Recently this variational method was improved ${ }^{17}$ by keeping the same periodic trial functions but now solving the GL equations iteratively; this iteration works much faster and allows to use many more Fourier coefficients (many thousands instead of only five in Ref. 15 ). I shall use this $2 \mathrm{D}$ iterative precision method of Ref. 17 for the calculation of the FLL at $B>0$. At low inductions $B \ll B_{c 2}$ this $2 \mathrm{D}$ method is supplemented by an iterative circular cell method presented in Appendix A. This 1D method yields accurate values of $h_{c 1}(\kappa)=H_{c 1} / H_{c 2}$, which then can be used in interpolation formulas. For convenience, I introduce the reduced fields $b=B / B_{c 2}, h=H / H_{c 2}, m=M / H_{c 2}$, such that one has $m=b-h, h_{c 1} \leq h \leq 1,0 \leq b \leq 1$, and $-h_{c 1} \leq m \leq 0$.

For completeness it should be mentioned that the isolated $\operatorname{vortex}^{18}$ and the FLL ${ }^{19}$ have also been com- 
b
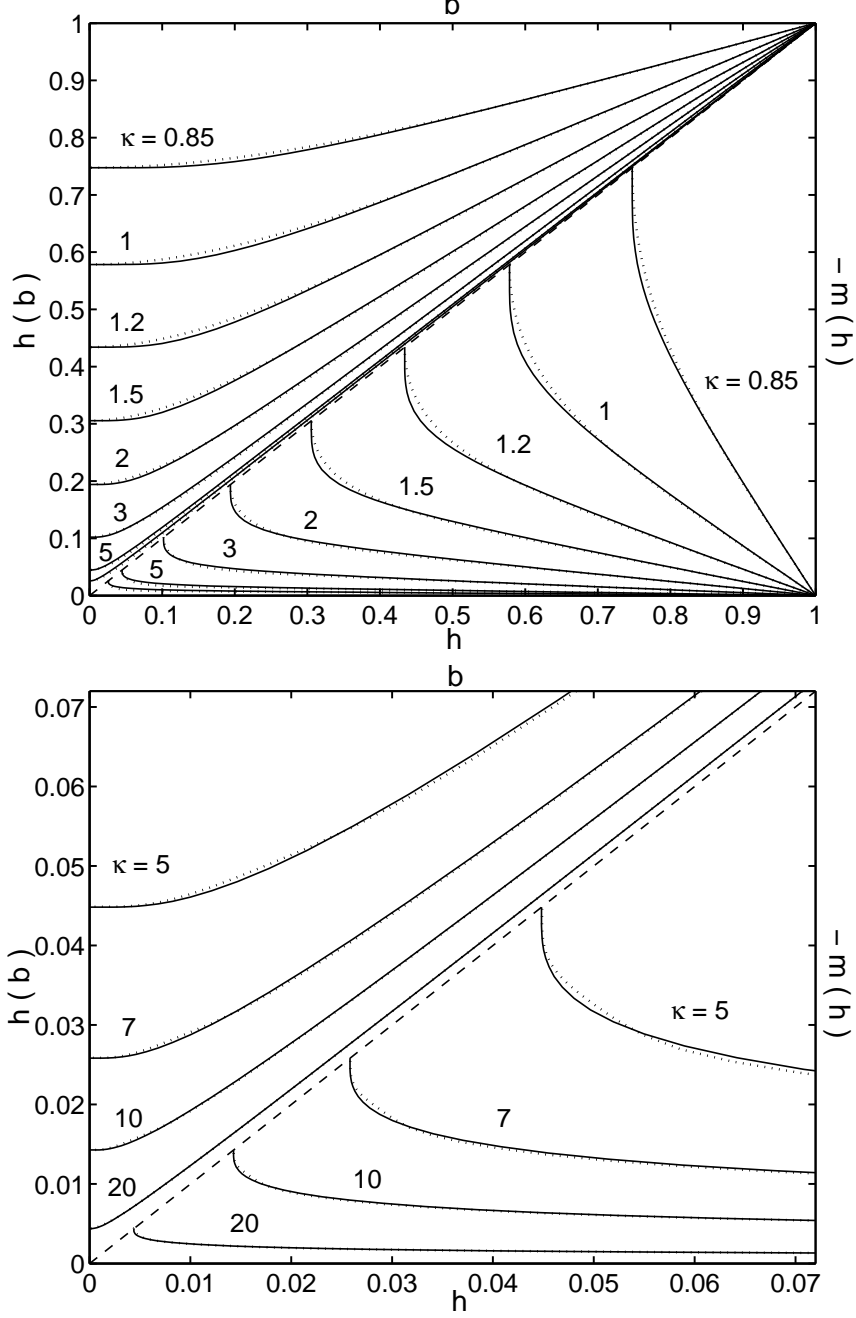

FIG. 1: Magnetization curves of the triangular FLL, which coincide within line thickness with the results for the square FLL and for the FLL obtained from the circular cell approximation, see Fig. 3 for the difference. Shown are $h=H / H_{c 2}$ versus $b=B / B_{c 2}$ (upper left triangle) and $-m=-M / H_{c 2}$ versus $h$ (lower right triangle). One has $m=b-h$. The lower panel shows an enlarged scale. The solid lines show the exact numerical result. The dotted lines show the simple interpolation Eq. (22) good for $\kappa \leq 5$ (upper panel), and the combined low and high field limit Eq. (23) good for $\kappa \geq 1$ (lower panel).

puted from BCS theory (valid at all temperatures) using the quasiclassical Eilenberger theory based on energyintegrated Green functions. This method was recently extended to compute the FLL structure and local density of states for s-wave $\frac{19.20 .21}{}$, d-wave ${ }^{21.22}$, and chiral pwave $^{23}$ superconductors. Very recently the GL method ${ }^{17}$ was generalized phenomenologically to lower temperatures and to charged vortices ${ }^{24}$.

\section{TRIANGULAR AND SQUARE FLUX-LINE LATTICES AND THE CIRCULAR CELL METHOD}

The properties of the FLL within GL theory are calculated by minimizing the GL free energy of the superconductor with respect to the complex GL function $\psi(\mathbf{r})$ and to the vector potential $\mathbf{A}(\mathbf{r})$ of the local magnetic field $\mathbf{B}(\mathbf{r})=\nabla \times \mathbf{A}$. In the usual reduced units.1.2.3.4.5.6.7 (length $\lambda$, magnetic field $\sqrt{2} H_{c}$, energy density $\mu_{0} H_{c}^{2}$, where $H_{c}$ is the thermodynamic critical field) the spatially averaged free energy $F$ of the GL theory referred to the Meissner state $(\psi=1, \mathbf{B}=0)$ reads

$$
F=\left\langle\frac{\left(1-|\psi|^{2}\right)^{2}}{2}+\left|\left(\frac{\nabla}{i \kappa}-\mathbf{A}\right) \psi\right|^{2}+\mathbf{B}^{2}\right\rangle .
$$

Here $\langle\ldots\rangle=(1 / V) \int d^{3} r \ldots$ means spatial averaging over the superconductor of volume $V$. Introducing the supervelocity $\mathbf{Q}(\mathbf{r})=\mathbf{A}-\nabla \varphi / \kappa$ and the magnitude $f(\mathbf{r})=|\psi|$ of $\psi(\mathbf{r})=f(\mathbf{r}) \exp [i \varphi(\mathbf{r})]$ one may express $F$ as a functional of the real and gauge invariant functions $f$ and Q,

$$
F=\left\langle\frac{\left(1-f^{2}\right)^{2}}{2}+\frac{(\nabla f)^{2}}{\kappa^{2}}+f^{2} Q^{2}+(\nabla \times \mathbf{Q})^{2}\right\rangle .
$$

In the presence of vortices $\mathbf{Q}(\mathbf{r})$ has to be chosen such that $\nabla \times \mathbf{Q}$ has the appropriate singularities along the vortex cores, see e.g. Eq.(B4) in App. B.

In this paper I consider the ideal periodic FLL in a homogeneous (pin-free) large superconductor in a uniform magnetic field $H$ along $z$. In this $2 \mathrm{D}$ situation one has $f=f(x, y), \mathbf{Q}=\mathbf{Q}(x, y)$, and $\mathbf{B}=\hat{\mathbf{z}} B(x, y)$. Within GL theory in reduced units the properties of this ideal FLL depend only on two parameters: the GL parameter $\kappa$ and the average induction $B=\langle B(x, y)\rangle$. The equilibrium magnetic field $H$, and the magnetization $M=B / \mu_{0}-H$, are obtained either from the definition $H=\partial F / \partial B$ or, more elegantly, from the virial theorem discovered by Doria, Gubernatis, and Rainer, ${ }^{25}$ which in reduced units reads

$$
H=\frac{\left\langle f^{2}-f^{4}+2 B(x, y)^{2}\right\rangle}{2 B} .
$$

Some of the properties of the FLL, and all properties of the isolated flux line, may be calculated in an elegant way by the circular cell approximation ${ }^{7.13 .14}$ as described in App. A. In the circular cell method the hexagonal Wigner-Seitz cell around each flux line is replaced by a circle with radius $R$ and same area $\pi R^{2}=\Phi_{0} / B$ if each flux line carries one quantum of flux $\Phi_{0}=h / 2 e=2.07 \cdot 10^{-5} \mathrm{Tm}^{2}$. In reduced units one has $\Phi_{0}=2 \pi / \kappa$ and $R / \lambda=R=\left(2 / b \kappa^{2}\right)^{1 / 2}$ with $b=B / B_{c 2}$. The boundary conditions on the CCM circle $r=R$ are $d f / d r=d B / d r=0$. I find that the free energy of the triangular FLL, $F_{t r}$, and its magnetization, $M_{t r}$, are reproduced by the CCM with high accuracy in the 
entire ranges of $\kappa$ and $B, 1 / \sqrt{2} \leq \kappa<\infty$ and $0 \leq b<1$. In particular, the CCM not only yields $H_{c 1}$ (in the limit $R \rightarrow \infty$ ) but it also reproduces the exact upper critical field $H_{c 2}(\kappa)$, and in the special case $\kappa=1 / \sqrt{2}$ even the exact result $H(B)=$ const $=H_{c}=H_{c 1}=H_{c 2}$. These somewhat surprising features of this approximation are related to the facts that $H_{c 2}$, and in the case $\kappa=1 / \sqrt{2}$ even the entire curve $H(B)$, are independent of the detailed arrangement of the flux lines, i.e., they are the same for triangular and square or honey-comb FLLs and for any other arrangement of single or multiple quanta flux lines. Another surprising finding is that the virial theorem, Eq. (3), works perfectly in the CCM. Figure 1 shows the magnetization curves $M(H)$ and the equilibrium field $H(B)$ of the superconductor obtained by the CCM for $\kappa=0.85,1,1.2,1.5,2,3,5,7,10$, and 20 .

In the limit $b \rightarrow 0$ the CCM yields the lower critical field $H_{c 1}$, which with high accuracy is fitted by the formula

$$
\begin{array}{r}
\mu_{0} H_{c 1}=\frac{\Phi_{0}}{4 \pi \lambda^{2}}[\ln \kappa+\alpha(\kappa)], \\
h_{c 1}=\frac{H_{c 1}}{H_{c 2}}=\frac{\ln \kappa+\alpha(\kappa)}{2 \kappa^{2}}, \\
\alpha(\kappa)=\alpha_{\infty}+\exp \left[-c_{0}-c_{1} \ln \kappa-c_{2}(\ln \kappa)^{2}\right] \pm \epsilon
\end{array}
$$

with $\alpha_{\infty}=0.49693, c_{0}=0.41477, c_{1}=0.775, c_{2}=$ 0.1303 , and $\epsilon \leq 0.00076$. This expression yields at $\kappa=$ $1 / \sqrt{2}$ the correct value $h_{c 1}=1$ and for $\kappa \gg 1$ it has the limit $\alpha=0.49693$. A simpler expression for $\alpha(\kappa)$, yielding an $h_{c 1}$ with error still less than $1 \%$ and with the correct limits at $\kappa=1 / \sqrt{2}$ and $\kappa \gg 1$, is

$$
\alpha(\kappa)=0.5+\frac{1+\ln 2}{2 \kappa-\sqrt{2}+2} .
$$

The CCM in principle cannot yield properties related to the different symmetries of the FLL, or to its shear modulus, and it cannot give the form factors (Fourier coefficients) of the magnetic field $B(x, y)$ that may be measured by neutron scattering. These subtle properties can be computed by the 2D method presented in Ref. 17 and in App. B. This effective numerical method expresses the smooth functions $f(x, y)^{2}$ and $B(x, y)$ as 2D Fourier series and determines the Fourier coefficients by iteration.

Figure 2 (top) shows the difference of the free energy densities of the triangular $\left(F_{t r}\right)$ and square $\left(F_{s q}\right)$ FLLs. This difference is proportional to the shear modulus $c_{66}$ of the triangular FLL (the shear modulus of the unstable square FLL is negative within GL theory) by the relation 17

$$
c_{66}=\left(3 \pi^{2} / 2\right)\left(F_{s q}-F_{t r}\right) .
$$

Note that this difference is very small, $0<\left(F_{s q}-\right.$ $\left.F_{t r}\right) /\left(\mu_{0} H_{c}^{2}\right)<0.0018$. Even smaller (by ten times) is the difference between the free energy densities of the CCM $\left(F_{c c}\right)$ and of the triangular FLL plotted in Fig. 2 (bottom). One has $0<\left(F_{c c}-F_{t r}\right) /\left(\mu_{0} H_{c}^{2}\right)<0.00020$.
This result shows that the CCM is an excellent approximation for global properties of the FLL. Both differences are largest for large $\kappa$ and have a maximum near $b \approx 0.3$. The finding $F_{s q}>F_{t r}$ means that the triangular FLL is stable for all $\kappa>1 / \sqrt{2}$. Note that for $\kappa=1 / \sqrt{2}$ one has exactly $F_{s q}=F_{c c}=F_{t r}=0$ for all $b$.

Figure 3 (top) shows the difference between the magnetizations $M_{s q}$ of the square FLL and $M_{t r}$ of the triangular FLL. Again, this difference is small, $0.0008<$ $\left.-\left(M_{s q}-M_{t r}\right) / H_{c 2}\right) \leq 0.00014$ and the relative difference has the limits $-0.018<\left(M_{s q}-M_{t r}\right) / M_{t r} \leq 0.0095$. Figure 3 (bottom) shows the difference between the magnetization $M_{c c}$ obtained by the CCM (see Fig. 1) and the exact value $M_{t r}$ of the triangular lattice. Like with the free energy, this difference is again smaller by a factor of ten than the difference between two lattice symmetries, $0.00016<-\left(M_{c c}-M_{t r}\right) / H_{c 2} \leq 0.00008$ and $-0.0011<\left(M_{c c}-M_{t r}\right) / M_{t r} \leq 0.0017$. The differences vanish exactly at $\kappa=1 / \sqrt{2}$, and also at $\kappa \rightarrow \infty$, since there $m=M / H_{c 2} \rightarrow 0$. The relative differences (insets in Fig. 3) are maximum at $\kappa \gg 1$.

The smallness of these differences explains why in Fig. 1 the magnetization curves for all three cases $M_{t r}$, $M_{s q}$, and $M_{c c}$ coincide within line thickness.

Figure 4 shows an example $(b=0.3, \kappa=1.5)$ comparing the spatial functions $f$ and $B$ of the triangular FLL and from the CCM. Shown are the cross sections $f(x, 0)$ along the nearest neighbor direction $x$ and $f(0, y)$ perpendicular to this, and $f(r)$ from the CCM $[a$ is the vortex spacing, $\left.a^{2} / \lambda^{2}=4 \pi /\left(\sqrt{3} b \kappa^{2}\right)\right]$. It is seen that $f(x, 0)$ and $f(r)$, and also $B(x, 0)$ and $B(r)$, coincide closely; at lower $b<0.3$ the difference is smaller than the line thickness. The solutions for the square FLL deviate more from the circular cell solutions.

The maximum, minimum, and saddle-point fields of the triangular FLL, $B_{\max }=B(0,0), B_{\min }=$ $B(0, a / \sqrt{3})$, and $B_{\text {sad }}=B(a / 2,0)$, depend on $b$ and $\kappa$. $B_{\max }$ is only slightly above the equilibrium field $H$, and $B_{\text {sad }}$ and $B_{\min }$ are close to each other and lie somewhat below the average field $B . \quad B_{\max }$ and $B_{\min }$ are shown in Fig. 3 of Ref. 17 as functions of $b$ for several $\kappa=0.707 \ldots 5$. In Fig. 5 the small differences $B_{\max }-H$, $B_{\text {sad }}-B$, and $B_{\text {sad }}-B_{\text {min }}$ are plotted versus $b$, in units $B_{c 2}$ and multiplied by a function of $\kappa$ such that the curves for all $\kappa \geq 1 / \sqrt{2}$ collapse at $b \rightarrow 1$. One finds for all $\kappa$ near $b=1$ :

$$
\begin{array}{r}
\frac{B_{\max }-H}{B_{c 2}} \approx 0.0351 \frac{\kappa^{2}-0.5}{\left(\kappa^{2}-0.069\right)^{2}}(1-b)^{2}, \\
\frac{B_{\text {sad }}-B}{B_{c 2}} \approx-0.146 \frac{1-b}{\kappa^{2}-0.069}, \\
\frac{B_{s a d}-B_{\min }}{B_{c 2}} \approx 0.0526 \frac{(1-b)}{\kappa^{2}-0.069} .
\end{array}
$$

The factor 0.069 in Eqs. (6)-(8) is $0.5-0.5 / \beta_{A}=0.0688$ where $\beta_{A}=1.1596$ is the Abrikosov parameter of the triangular FLL. Plots of $B_{c c}(R)-B_{\min }$ where $B_{c c}(R)$ is the field value at the boundary of the circular cell in the 

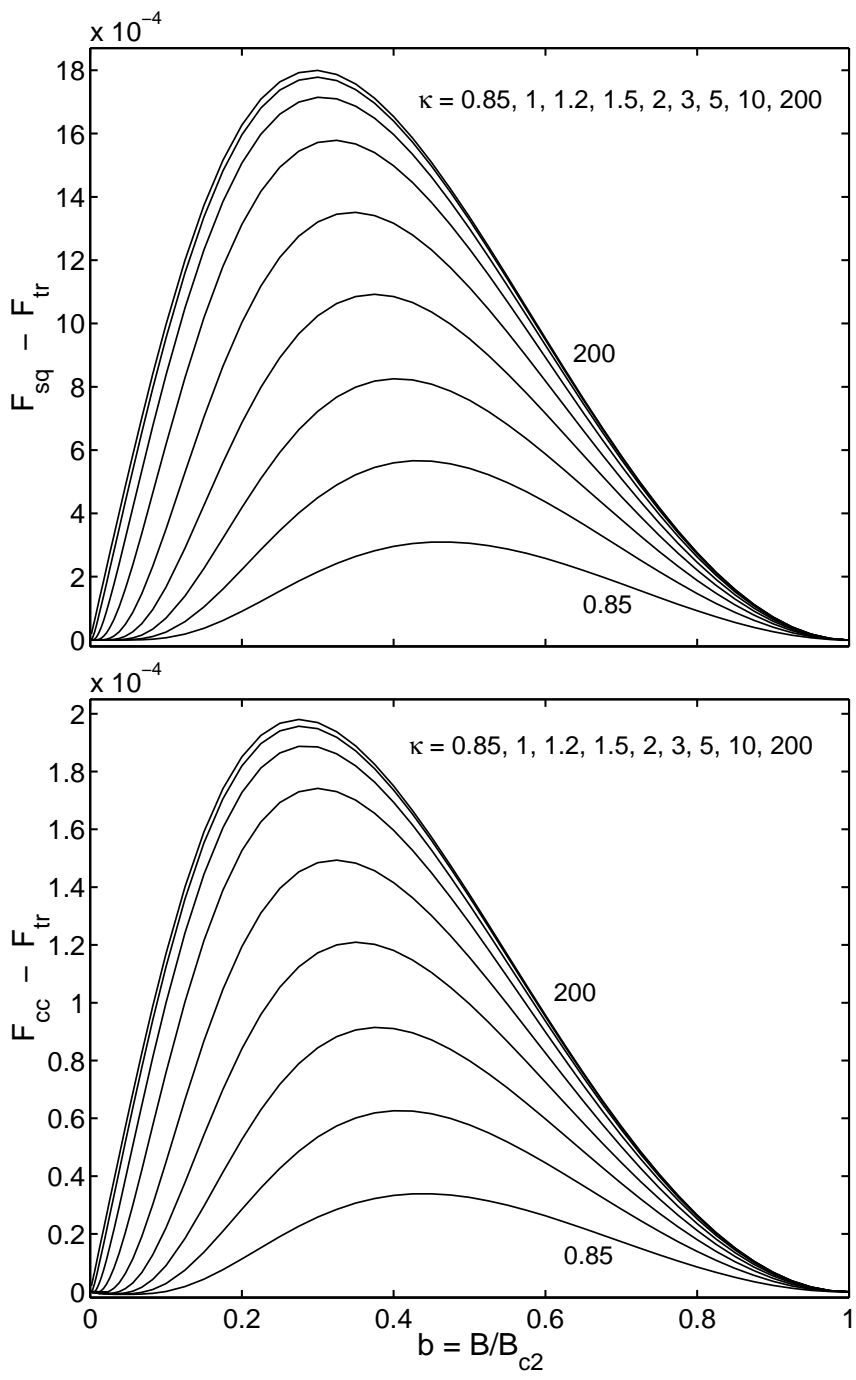

FIG. 2: Top: The difference of the free energy densities of the triangular $\left(F_{t r}\right)$ and square $\left(F_{s q}\right)$ FLLs in units $\mu_{0} H_{c}^{2}$, plotted versus the reduced induction $b=B / B_{c 2}$ for $\kappa=0.85$, $1,1.2,1.5,2,3,5,10$, and 200. This difference equals $\left(2 / 3 \pi^{2}\right)=0.068$ times the shear modulus $c_{66}$ of the triangular FLL. Bottom: The very small difference between the free energy densities of the circular cell method $\left(F_{c c}\right)$ and of the triangular FLL. Note that the top and bottom plots look similar, but the scales of the ordinate differ by a factor of about ten.

CCM, look similar to the plots of $B_{\text {sad }}-B_{\text {min }}$ in Fig. 5 (lower panel), since the value $B_{c c}(R)$ lies approximately in the middle between $B_{\min }$ and $B_{\text {sad }}$, see Fig. 4. Since for $\kappa \gg 1$ and $b \ll 1 / \kappa^{2}$ the field in the vortex center equals $B_{\max }=2 H_{c 1}$, one has $B_{\max }-H \rightarrow H_{c 1}$, and thus the function plotted in Fig. 5 (upper panel) for $b \rightarrow 0$ tends to the limit $\left(b_{\max }-h\right) \times \kappa^{2} \rightarrow h_{c 1} \kappa^{2} \approx \frac{1}{2}(\ln \kappa+$ $0.50)$, cf. Eq. (4).

The variance of the magnetic field is

$$
\sigma=\left\langle[B(x, y)-B]^{2}\right\rangle=\left\langle B(x, y)^{2}-B^{2}\right\rangle=\sum_{\mathbf{K} \neq 0} B_{\mathbf{K}}^{2},
$$
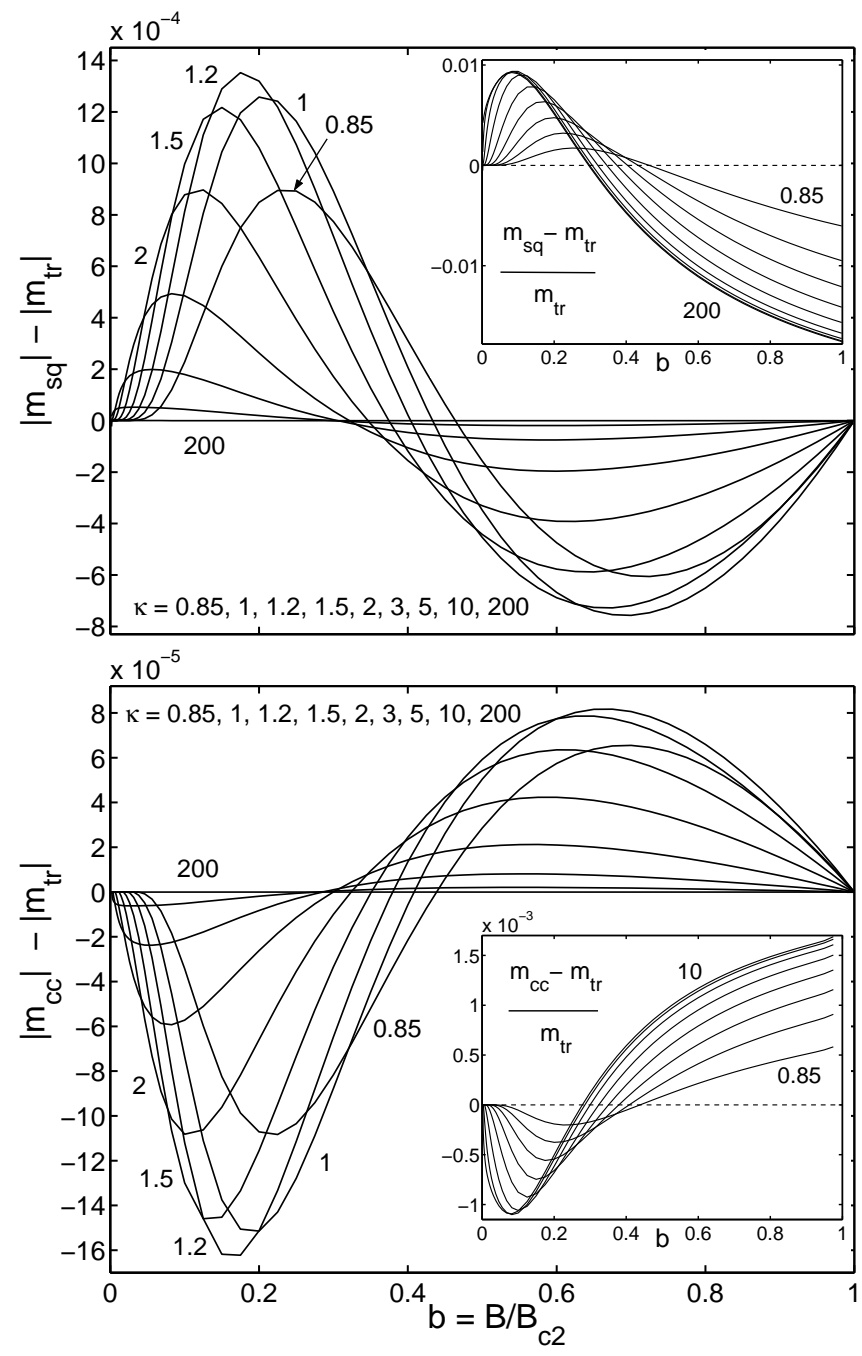

FIG. 3: Top: The difference between the magnetizations $M_{s q}$ of the square FLL and $M_{t r}$ of the triangular FLL in units $H_{c 2}$, plotted versus the reduced induction $b=B / B_{c 2}$ for $\kappa=0.85,1,1.2,1.5,2,3,5,10$, and 200. The inset shows the relative difference. Bottom: The difference between the magnetization $M_{c c}$ obtained by the CCM (see Fig. 1) and the exact value $M_{t r}$ of the triangular lattice. The inset shows the relative difference.

where $B_{\mathbf{K}}$ are the Fourier coefficients of $B(x, y)=$ $\sum_{\mathbf{K}} B_{\mathbf{K}} \cos \mathbf{K r}$ and $\mathbf{K}$ the vectors of the reciprocal lattice of the FLL (App. B). Near $b=1$ the Abrikosov solution of the linearized GL theory ${ }^{11,26}$ yields for all $\kappa$ values ${ }^{27}$

$$
\begin{gathered}
\sigma=7.52 \cdot 10^{-4} \frac{\Phi_{0}^{2}}{\lambda^{4}} \frac{\kappa^{4}(1-b)^{2}}{\left(\kappa^{2}-0.069\right)^{2}}, \\
S \equiv \frac{\sqrt{\sigma}}{B_{c 2}}=0.172 \frac{1-b}{\kappa^{2}-0.069} .
\end{gathered}
$$

The functions $S$ and $S /(1-b)$ are plotted in Fig. 6 versus $\sqrt{b}$ for various $\kappa$. It can be seen that Eq. (10) is a rather good approximation for the large range $0.25<b<1$. At smaller $b$ the variance $\sigma(b)$ has a maximum and then goes to zero again at $b=0$. 


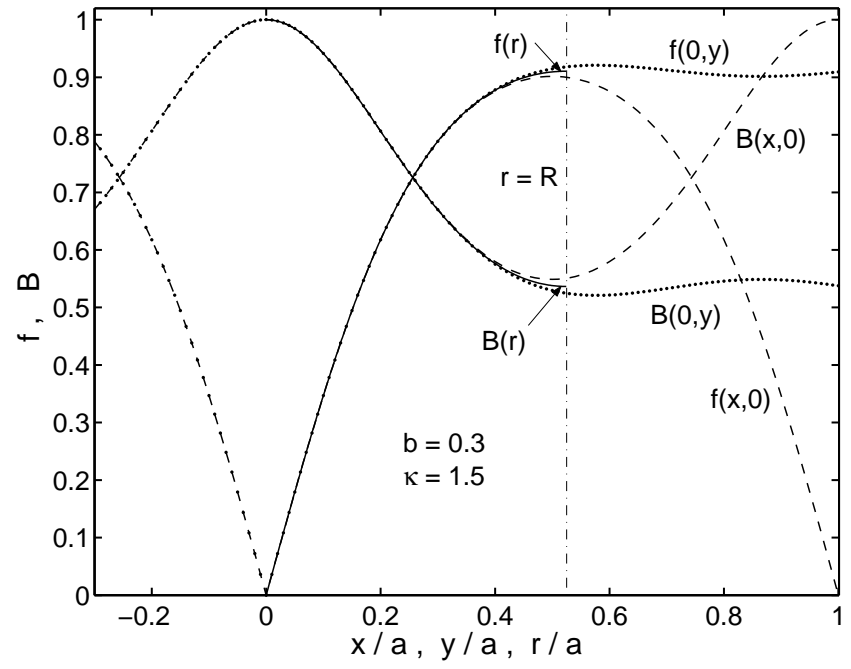

FIG. 4: Comparison of the GL functions $f$ and magnetic inductions $B$ calculated for the triangular FLL and from the circular cell approximation for the example $b=0.3, \kappa=1.5$. Shown are the cross sections $f(x, 0), B(x, 0)$ along the nearest neighbor direction $x$, and $f(0, y), B(0, y)$ along the perpendicular direction $y$, and $f(r), B(r)$ from the CCM. All $B$ are in units $B(0,0)$ of the triangular FLL. Small deviations can be seen only close to the cell boundary $r=R$, $R / a=3^{1 / 4}(2 \pi)^{-1 / 2}=0.525$. At lower $b$ the deviations are even smaller.

For small inductions $b \ll 1$ and large $\kappa$ one can use the London approximation $B_{\mathbf{K}}=B /\left(1+K^{2} \lambda^{2}\right)$. For the appropriate cutoff at large magnitudes $K \sim \xi^{-1}=\kappa / \lambda$ see Ref. 28, 29 and below. In the range $0.13 / \kappa^{2} \ll b \ll 1$ the unity in the denominator of $B_{\mathbf{K}}$ may be disregarded since $K^{2} \lambda^{2} \geq(4 \pi / \sqrt{3}) b \kappa^{2}=7.255 b \kappa^{2}$. Thus $B$ drops out and $\sigma$ becomes independent of $b b^{27}$

$$
\sigma=0.00371 \frac{\Phi_{0}^{2}}{\lambda^{4}}, \quad S=\frac{0.383}{\kappa^{2}} .
$$

This often used formula corresponds to the upper axis in Fig. 6. One can see that this approximation is good only for very large $\kappa \geq 70$ and only in the range of $b$ near the maximum of $\sigma$. At very small $b \ll 0.13 / \kappa^{2}$ both $\sigma(b)$ and $S(\sqrt{b})$ drop linearly to zero when $b \rightarrow 0$. In this range the sum in Eq. (9) can be evaluated as an integral, yielding

$$
\sigma=\frac{b \kappa^{2}}{8 \pi^{2}} \frac{\Phi_{0}^{2}}{\lambda^{4}}, \quad S=\frac{\sqrt{b / 2}}{\kappa} .
$$

This approximation is good for $\kappa \geq 5$ and very small $b$ $\left(b<0.01 / \kappa^{2}\right.$ for $\kappa=5 ; b<0.04 / \kappa^{2}$ for $\left.\kappa \geq 10\right)$, see the two straight lines in Fig. 6. For $\kappa \geq 5$ a good approximation, with less than $5 \%$ error from $b=1$ down to the value $b \approx 0.25 / \kappa^{1.3}$ where the maximum of $\sigma$ occurs, is

$$
S \equiv \frac{\sqrt{\sigma}}{B_{c 2}} \approx 0.172 \frac{1-b}{\kappa^{2}}\left[1+1.21(1-\sqrt{b})^{3}\right] .
$$

This approximation is much better that the interpolation, Eq. (3) of Ref. 27.

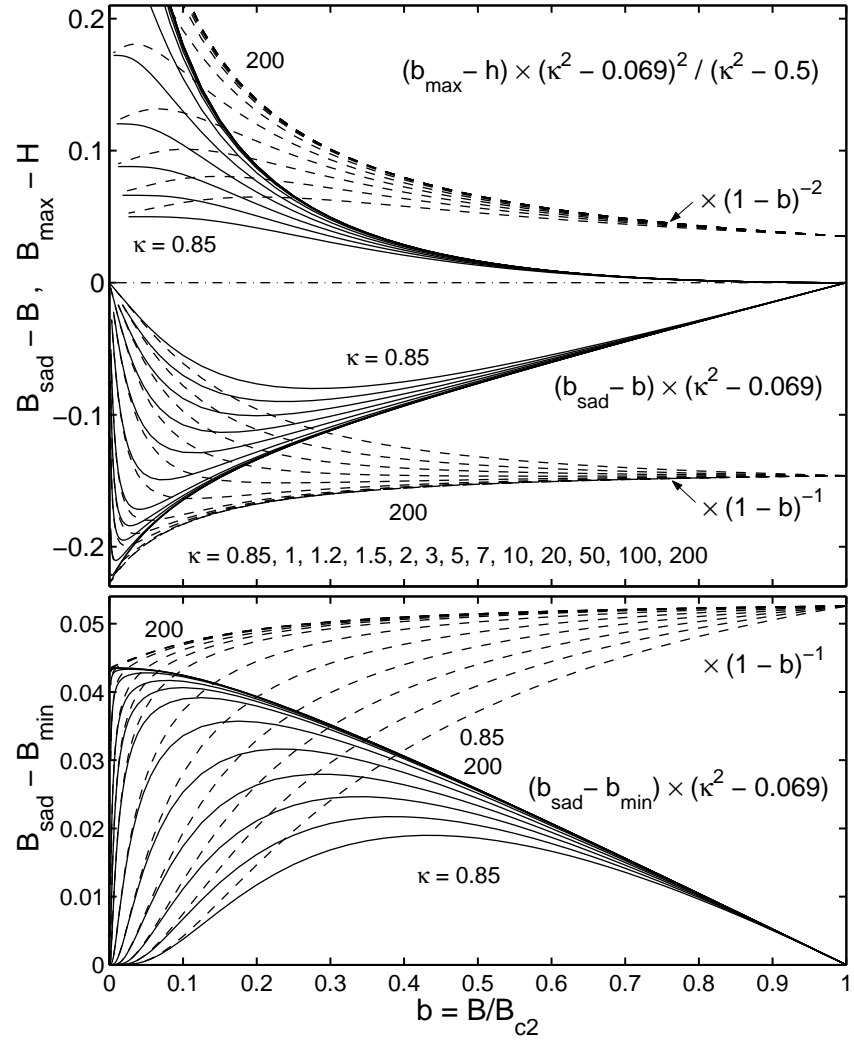

FIG. 5: Maximum field $B_{\max }=b_{\max } B_{c 2}$ minus applied field $H$, saddle-point field $B_{\text {sad }}=b_{\text {sad }} B_{c 2}$ minus induction $B$, and $B_{\text {sad }}$ minus minimum field $B_{\min }=b_{\min } B_{c 2}$, for the triangular FLL, plotted versus $b=B / B_{c 2}$ for $\kappa=0.85,1,1.2,1.5,2$, $3,5,7,10,20,50,100,200$. The solid lines show these small differences in units $B_{c 2}$, multiplied by appropriate functions of $\kappa$ to obtain collapse of the curves near $b=1$. The dashed lines show the same functions multiplied by factors $(1-b)^{-2}$ and $(1-b)^{-1}$ such that they tend to a finite constant value near $b=1$, cf. Eqs. (6) - (8).

\section{MAGNETIZATION CURVES}

This section presents analytic expressions which approximate the computed magnetization $m=M / H_{c 2}=$ $b-h$ (Fig. 1) as a function of the induction $b=B / B_{c 2}$ or of the thermodynamic field $h=H / H_{c 2}$. We distinguish approximations working at high or low inductions.

\section{A. Approximation for high inductions}

The linearized GL theory yields for $1-b \ll 1$ Abrikosov's $B_{c 2}$ solution ${ }^{1,11}$

$$
m \approx m_{A}=-\frac{1-b}{\left(2 \kappa^{2}-1\right) \beta_{A}+1}
$$

where $\beta_{A}=\left\langle\omega_{A}^{2}\right\rangle /\left\langle\omega_{A}\right\rangle^{2}=1+\sum_{m, n} \exp \left[K_{m n}^{2} S /(4 \pi)\right]$ (Ref. 11,30 and App. B) is the Abrikosov parameter, $\beta_{A}=1.1595953\left(\beta_{A}=1.1803406\right)$ for the triangular 


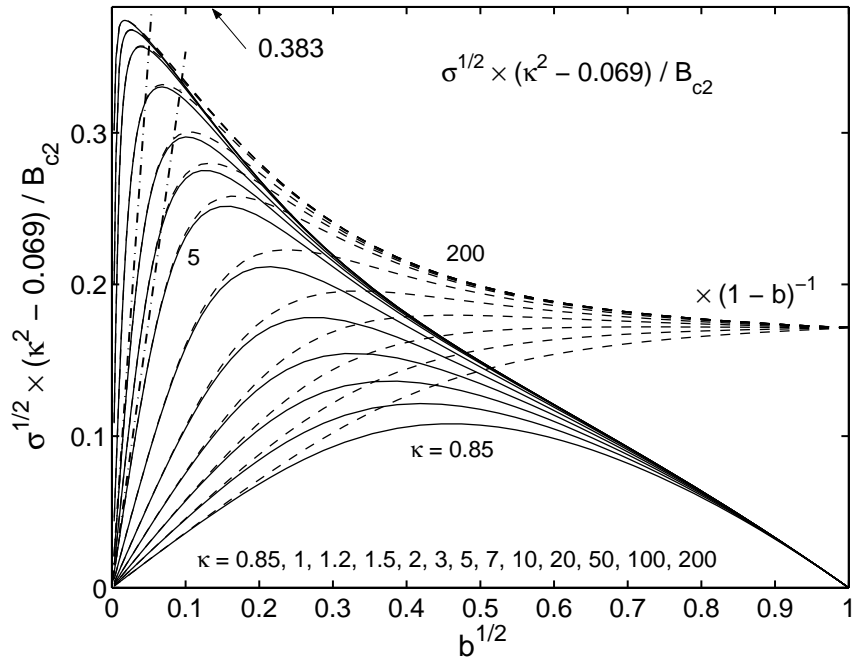

FIG. 6: The magnetic field variance $\sigma=\left\langle[B(x, y)-B]^{2}\right\rangle$ of the triangular FLL for $\kappa=0.85,1,1.2,1.5,2,3,5,7,10,20$, 50, 100, 200 plotted in units of $B_{c 2}$ as $\sqrt{\sigma} \cdot\left(\kappa^{2}-0.069\right) / B_{c 2}$ (solid lines) such that the curves for all $\kappa$ collapse near $b=1$, cf. Eq. (10). The dashed lines show the same functions divided by $(1-b)$ such that they tend to a finite constant 0.172 at $b=1$. All curves are plotted versus $\sqrt{b}=\sqrt{B / B_{c 2}}$ to stretch them at small $b$ values and show that they go to zero linearly. The limits, Eq. (12), for $\kappa=5$ and $\kappa=10$ are depicted as dash-dotted straight lines. The upper frame 0.383 shows the approximation (11).

(square) FLL. The linear magnetization $m_{A}(b, \kappa)$ is a good approximation in the range $0.5 \leq b \leq 1$, see Fig. 1 . This suggests the following fit to the exact $m$ :

$$
\begin{aligned}
& m(b, \kappa)=m_{A}-(1-b)^{2} \exp \left[f_{1}(b)\right] g_{1}(\kappa)+\epsilon_{1}, \\
& f_{1}(b)=2.50 u^{2}-8.08 u+0.39, u=(1-b)^{0.41} \\
& g_{1}(\kappa)=\left(1.133+1.926 / \kappa^{2.25}\right)\left(2 \kappa^{2}-1\right) /\left(2 \kappa^{4}\right)
\end{aligned}
$$

with relative error $\left|\epsilon_{1} / m\right|<0.0013$ for $b>0.5$ for the triangular FLL. Formula (15) is a good approximation with relative error $<1 \%$ for all $\kappa$ in the large range of fields $1 /\left(4 \kappa^{2}\right)+5 \cdot 10^{-4} \leq b \leq 1$, see Fig. 7 .

The same expression (14) fits also the $m(b, \kappa)$ of the square FLL, with somewhat larger error if the same functions $f_{1}(b), g_{1}(\kappa)$ are used rather than the optimally fitted ones. For the difference $m_{\mathrm{tr}}-m_{\mathrm{sq}}$ see Fig. 3 .

\section{B. Approximation for "intermediate fields"}

For completeness I mention here also the London approximation ${ }^{3}$ which was supposed to be good in the "intermediate field range" $H_{c 1} \ll H \ll H_{c 2}$ that exists only in superconductors with extremely large $\kappa$. Within London theory the induction is (see App. B)

$$
B(x, y)=B \sum_{\mathbf{K}} \frac{\cos \mathbf{K r}}{1+K^{2} \lambda^{2}},
$$
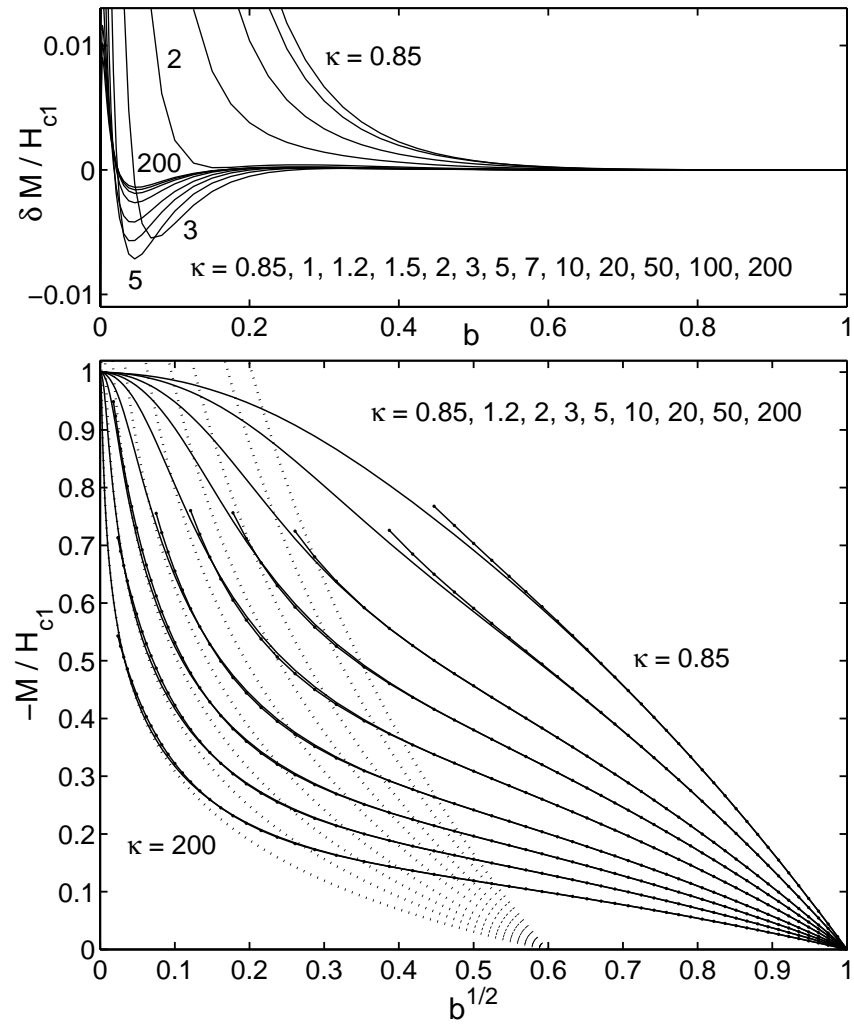

FIG. 7: Lower panel: The exact magnetization $M$ of the triangular FLL (solid lines) and the fit, Eq. (15), (solid lines with dots) plotted for many $\kappa$ values versus $\sqrt{b}=\sqrt{B / B_{c 2}}$ to stretch the low field region. Shown is $-M$ normalized to its maximum value $H_{c 1}$ occurring at $b=0$. The fit (15) is good for all $\kappa$ and not too small $b>1 /\left(4 \kappa^{2}\right)+0.0005$. Upper panel: The deviation $\delta M$ of the fit from the exact $M$ is very small when $b>0.5$. The dotted lines in the lower panel show the old London approximation, Eq. (18).

where the sum goes over all $K$-vectors with length from $K=0$ to some cutoff $K \approx \xi^{-1}$. Inserting this into the London free energy density $\left[B(\mathbf{r})^{2}+\lambda^{2}(\nabla \times \mathbf{B})^{2}\right] /\left(2 \mu_{0}\right)$ and averaging over the superconductor one gets

$$
F=\sum_{\mathbf{K}} \frac{\left(B^{2} / 2 \mu_{0}\right)}{1+K^{2} \lambda^{2}} \approx \frac{B^{2}}{2 \mu_{0}}+\frac{B \Phi_{0}}{2 \mu_{0}} \int \frac{d^{2} k}{4 \pi^{2}} \frac{1}{k^{2} \lambda^{2}} .
$$

The integral from $k_{\min }^{2} \approx\left(K_{10} / 2\right)^{2} \approx \pi^{2} B / \Phi_{0}$ to $k_{\max }^{2} \approx$ $\xi^{-2}=2 \pi B_{c 2} / \Phi_{0}$ equals $\left(4 \pi \lambda^{2}\right)^{-1} \ln \left(\gamma^{\prime} / b\right)$ where $\gamma^{\prime}$ is some constant and $b=B / B_{c 2}$ as above. This yields

$$
\begin{aligned}
-M & =H-\frac{B}{\mu_{0}}=\frac{\partial F}{\partial B}-\frac{B}{\mu_{0}}=\frac{\Phi_{0}}{8 \pi \lambda^{2} \mu_{0}} \ln \frac{\gamma}{b} \\
-m & =\frac{-M}{B_{c 2}}=\frac{1}{4 \kappa^{2}} \ln \frac{0.358}{b}
\end{aligned}
$$

with constant $\gamma=\gamma^{\prime} / e=0.3575 \ldots$ obtained by our fit to the numerical $m(b)$ at $\kappa=200$. This old London approximation is shown in Fig. 7 as dotted lines. One sees that this fit works only at large $\kappa \geq 20$ in the relatively small interval $1 /\left(2 \kappa^{2}\right) \leq b \leq 0.01$, i.e. at very low $b$ (but 


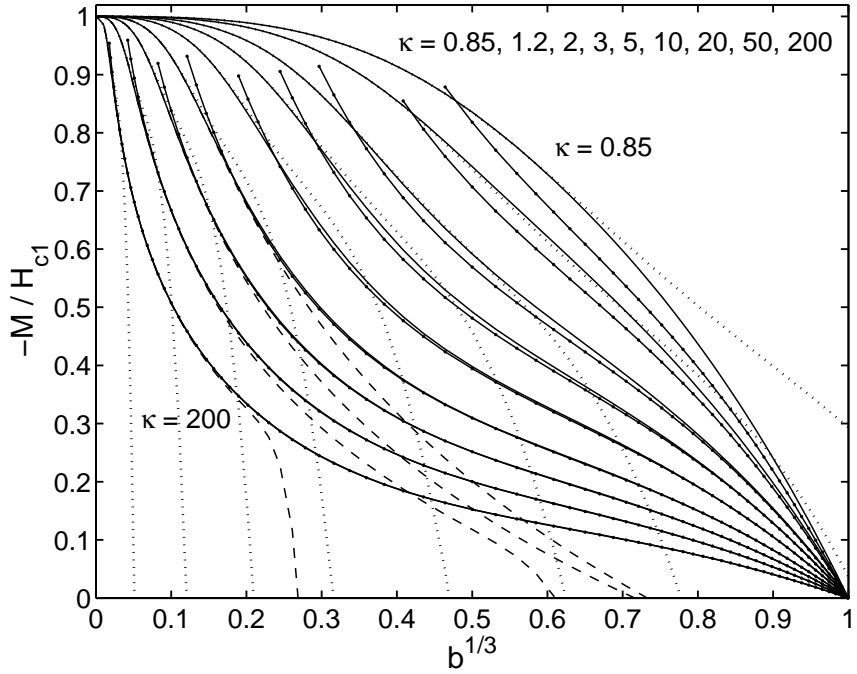

FIG. 8: Exact magnetization of the triangular FLL (solid lines) and the logarithmic fit, Eq. (19), (solid lines with dots) plotted versus $b^{1 / 3}$ to stretch the region at small $b=B / B_{c 2}$. The dotted lines show the London nearest-neighbor approximation, Eq. (20). The dashed lines show the London expression, Eq. (C6), with the sum taken over all shells up to $\nu_{\max }=100$ vortex spacings. Both London approximations are good fits at very low $b$ and all $\kappa$.

not too low $b)$. It gives $m=0$ at $b=\gamma$ for all $\kappa$. This fit is slightly improved by replacing $\ln (\gamma / b)$ by $\ln (1-\gamma+\gamma / b)$, which gives the correct $m=0$ at $b=1$.

A much better fit in the spirit of this logarithmic approximation is (see Fig. 8)

$$
\begin{aligned}
-m & =\frac{1}{4 \kappa^{2}} \ln \left[1+\frac{1-b}{b} f_{2}(b)\right], \\
f_{2}(b) & =0.357+2.890 b-1.581 b^{2} .
\end{aligned}
$$

This fit is good for $\kappa \geq 3$ (error $<3 \%$ ) and $\kappa \geq 5$ (error $<1 \%$ ) in the large ranges $(\ln \kappa+1) /\left(10 \kappa^{2}\right) \leq b \leq 1$ for $\kappa=3 \ldots 200$. These intervals of validity may also be expressed as $-M / H_{c 1}=-m / h_{c 1} \leq 0.8(0.85)$ for $\kappa \leq 20$ $(\kappa \geq 50)$.

\section{Approximation for low inductions}

All the above approximations do not describe the correct vertical slope of $M(H)$ at $H=H_{c 1}$, or zero slope of $H(B)$ and unity slope of $M(B)=B-H$ at $B=0$. This is achieved by the London approximation of pairwise interacting vortices described in App. C. For very small $b \ll 1$ one may account only for the nearest neighbor shell of six vortices in the triangular FLL of spacing $a=c \lambda$. With $h(b)$, Eq. (C8), this yields for $-m(b)=h(b)-b$ :

$$
-m \approx h_{c 1}-b+\frac{3 \sqrt{\pi c}}{2 \kappa^{2}} e^{-c}\left[1+\frac{19}{8 c}-\frac{47}{128 c^{2}}\right],
$$

$$
c=\frac{a}{\lambda}=\left(\frac{4 \pi / \sqrt{3}}{b \kappa^{2}}\right)^{1 / 2} .
$$

Formula (20) correctly describes the steep diverging slope of $m(h) \rightarrow \infty$ or slopes $m(b)^{\prime} \rightarrow 1$ and $h(b)^{\prime} \rightarrow 0$ as $b \rightarrow 0$ and is valid for $0 \leq b \leq 2.5 / \kappa^{2}$ for $\kappa \geq 7$, Accidentally it also fits well $m(b)$ for $\kappa \leq 2$ and $b \leq 0.2$, see the dotted lines in Fig. 8. A smoother fit is obtained by the exact London expression (C6) if one or three neighbor shells are included in the sum. But taking more terms in the sum improves the fit only at large $\kappa$. Accounting for neighbors up to $\nu=100$ lattice spacings apart (about 5000 terms) one gets good approximation to $m$ and $h$ for $0 \leq b \leq 0.01$ $(0.02,0.05)$ if $\kappa \geq 20(\kappa=7, \kappa=2)$, see Fig.8. In the limit $\nu \rightarrow \infty$ the infinite sum (C6) reproduced Eq. (18), i.e, the dashed curves in Fig. 8 for $\kappa=50,200$ then will straighten and cut the axis $M=0$ at $b=\gamma=0.358$ $\left(b^{1 / 2}=0.60\right.$ in Fig. $7, b^{1 / 3}=0.71$ in Fig. 8$)$.

\section{General interpolation}

All the approximations for $m(b)$ and $h(b)$ known so far, including the above formulas, fit either the low or high field regions. The formulas (15) and (20) [or better, Eq. (C6) with the sum taken over three shells] have a small overlap for all $\kappa$ and thus, together, they fit the entire range $0 \leq b \leq 1$ [though the good fit of the low- $\kappa$ data by the London expression (20) or (C6) is accidental].

For practical purposes one may construct interpolation formulas that approximate the numerically obtained magnetization in the entire range $0<b<1$. They should satisfy the five conditions

$$
\begin{gathered}
h(0)=h_{c 1}, h^{\prime}(0)=0, h(1)=1, h^{\prime}(1)=1-p, \\
h^{\prime \prime}(1)=0, \quad p=m^{\prime}(1)=\left[\left(2 \kappa^{2}-1\right) \beta_{A}+1\right]^{-1},
\end{gathered}
$$

with $h_{c 1}(\kappa)$ from Eq. (4). A simple expression that satisfies all these conditions is

$$
-m(b, \kappa)=h-b=p(1-b)+\left(h_{c 1}-p\right)(1-b)^{\eta}
$$

with $\eta(\kappa)=(1-p) /\left(h_{c 1}-p\right)$. Formula (22) approximates the exact $-m(b)$ well for $\kappa \leq 2$ with relative deviation $|\epsilon|<3 \%$, for $\kappa=3$ with $-2 \%<\epsilon<6 \%$, and for $\kappa=5$ with $-1 \%<\epsilon<16 \%$, see the dotted lines in Fig. 1, top.

For large $\kappa$, general interpolation formula are more difficult to construct because of the nonanalytic limiting expression Eq. (20). One may, however, combine the $m_{\text {low }}$ from Eq. (20) with the $m_{\text {high }}$ from Eq. (19) using a smooth transition at $b \approx\left(2 \kappa^{2}\right)^{-1}$, e.g., with weights $1-w$ and $w=\frac{1}{2}+\frac{1}{2} \tanh \left[2.5\left(2 b \kappa^{2}-1\right)\right]$, or slightly better, $w=\frac{1}{2}+\frac{1}{2} \operatorname{erf}\left[2\left(2 b \kappa^{2}-1\right)\right]$, yielding

$$
m(b, \kappa)=(1-w) m_{\text {low }}+w m_{\text {high }} .
$$

This interpolation between expressions (19) and (20) works well for $0<b<1$ with relative error $|\epsilon|<2 \%$ for $\kappa \geq 5$ and $-3.5 \%<\epsilon<2 \%$ for $\kappa \geq 1$, see the dotted lines in Fig. 1 (bottom). Thus, $m$ in the entire ranges of $b$ and $\kappa$ may be approximated by Eq. (22) or Eq. (23). 


\section{CONCLUSION}

The properties of the ideal periodic flux-line lattice in superconductors are calculated from Ginzburg-Landau theory for the entire ranges of GL parameters $1 / \sqrt{2} \leq$ $\kappa<\infty$ and inductions $0 \leq b=B / B_{c 2}<1$. The differences between the free energies and magnetizations of the triangular and square vortex lattices and the values obtained by the circular cell approximation are investigated in detail. Approximate analytical expressions are given for the variance $\sigma(b, \kappa)$ of the periodic induction and for the magnetization $m(b, \kappa)$. These limiting and interpolation formulas should replace previous approximate expressions that have rather limited validity.

The numerical methods presented in the appendices, in principle may be applied also to theories going beyond the isotropic GL theory considered here.

\section{APPENDIX A: ISOLATED VORTEX AND CIRCULAR CELL METHOD}

The calculation of the isolated flux line and of the FLL within the circular cell method, is a cylindrically symmetric problem. The free energy depends on the magnitude of the GL function $f(r)$ and on the magnetic induction $B(r)$ (along $z$ ) related to the vector potential $A(r)$ and supervelocity $Q(r)$ (along $\varphi$ ) by

$$
B=\frac{(A r)^{\prime}}{r}=\frac{(Q r)^{\prime}}{r}, \quad Q=A-\frac{1}{\kappa r} .
$$

In reduced units $\sqrt{2} H_{c}=\mu_{0} H_{c}^{2}=\lambda=1$, the free energy of a flux line or of the circular cell with radius $R$ $\left(\pi R^{2}=\Phi_{0} / B\right)$ averaged over this cell and referred to the Meissner state $(f=1, B=0)$ reads

$$
F_{c c}=\int_{0}^{R}\left[\frac{\left(1-f^{2}\right)^{2}}{2}+\frac{\left(f^{\prime}\right)^{2}}{\kappa^{2}}+f^{2} Q^{2}+B^{2}\right] \frac{2 \pi r d r}{\pi R^{2}}
$$

with $f^{\prime}=d f / d r$. Minimizing the functional (A2) with respect to $f(r)$ and $Q(r)$ we obtain the two GL equations, which may be written in the form

$$
\begin{array}{r}
-f^{\prime \prime}+\kappa^{2} f=\kappa^{2}\left(2 f-f^{3}-Q^{2} f\right)+f^{\prime} / r, \\
B^{\prime}=f^{2} Q=j,
\end{array}
$$

where $j=B^{\prime}$ is the current density. In Eq. (A3) a term $\kappa^{2} f$ was added on both sides to improve the convergence of the iteration below. The boundary conditions are

$$
f(0)=f^{\prime}(R)=j(0)=j^{\prime}(R)=0 .
$$

An appropriate ansatz in terms of Fourier series is

$$
\begin{aligned}
& f(r)=\sum_{m=1}^{M} f_{G} \sin G r, \quad G=\frac{\pi(2 m-1)}{2 R}, \\
& A(r)=\sum_{n=1}^{N} a_{K} \sin K r+\frac{r}{2} B, \quad K=\frac{\pi n}{R},
\end{aligned}
$$

$$
\begin{array}{r}
B(r)=\sum_{n=1}^{N} a_{K} \frac{\sin K r+K r \cos K r}{r}+B, \\
Q(r)=\sum_{n=1}^{N} a_{K} \sin K r-\frac{1-r^{2} / R^{2}}{\kappa r}, \\
j(r)=\sum_{n=1}^{N} a_{K} \frac{K r \cos K r-\left(1+K^{2} r^{2}\right) \sin K r}{r^{2}} .
\end{array}
$$

For the equidistant grid $r_{i}=\left(i-\frac{1}{2}\right) R / N_{r}, i=1,2, \ldots N_{r}$, one has the orthogonality relation

$$
\sum_{i=1}^{N_{r}} \sin G r_{i} \sin G^{\prime} r_{i}=\frac{1}{2} N_{r} \delta_{G G^{\prime}}
$$

and similar equations for $\sin K r_{i}$ and $\cos K r_{i}$. The GL equations (A3) and (A4) thus may be written in the form of equations for the Fourier coefficients $f_{G}$ and $a_{K}$ :

$$
\begin{array}{r}
f_{G}=\frac{1}{G^{2}+\kappa^{2}} \frac{2}{N_{r}} \sum_{i=1}^{N_{r}} \sin G r_{i} \times \\
{\left[\kappa^{2}\left(2 f-f^{3}-Q^{2} f\right)+f^{\prime} / r_{i}\right],} \\
a_{K}=\frac{1}{K^{2}+1}\left[a_{K}+\frac{2}{N_{r}} \sum_{i=1}^{N_{r}} \sin K r_{i} \times\right. \\
\left.\left(\sum_{n^{\prime}=1}^{N} a_{K^{\prime}} \frac{K r \cos K r-\sin K r}{r^{2}}-f^{2} Q\right)\right] .
\end{array}
$$

These two equations may be used to obtain the $f_{G}$ and $a_{K}$ by iteration, starting with appropriate initial values. The iteration becomes more stable and faster if the value of the previous iteration step is added with a certain weight $(1-c)<1$, e.g., $\mathrm{c}=0.6$, according to the algorithm:

$$
\begin{aligned}
& f_{G} \leftarrow(1-c) f_{G}+c F_{G}\{f, Q\}, \\
& a_{K} \leftarrow(1-c) a_{K}+c A_{K}\{f, Q\},
\end{aligned}
$$

with the symbols $F_{G}\{f, Q\}$ and $A_{K}\{f, Q\}$ denoting the right hand sides of Eqs. (A12) and (A13), respectively. Rapid convergence is achieved by iterating equations (A14), (A15) alternately. The equilibrium magnetic field $H$ is then obtained from Eq. (3), and the magnetization from

$$
M=\frac{2}{B R} \int_{0}^{R}\left[\frac{f^{4}-f^{2}}{2}+B^{2}-B(r)^{2}\right] r d r .
$$

At very large $\kappa$ and very small $b$ a large number $N_{r}$ of grid points $r_{i}$ is needed to achieve high accuracy, $N_{r} \gg R / \xi=R \kappa=\sqrt{2 / b}$. In this case the accuracy with a limited number of grid points may be improved by choosing a nonequidistant grid, e.g., $r_{i}=u_{i}^{2}$ with equidistant $u_{i}=\left(i-\frac{1}{2}\right) \sqrt{R} / N_{r}$. To use the orthogonality relations one then has to express $f, B$, and $Q$ as Fourier series in the new variable $u=r^{2}$ and also write the 
two GL equations in terms of the variable $u$, using, e.g., $f^{\prime}(r)=f^{\prime}(u) / 2 u$ and $f^{\prime \prime}(r)=f^{\prime \prime}(u) / 4 u^{2}-f^{\prime}(u) / 4 u^{3}$. This yields

$$
\begin{array}{r}
f^{\prime \prime}(u)=4 u^{2} \kappa^{2}\left(-f+f^{3}+Q^{2} f\right)+f^{\prime} / u, \\
B^{\prime}(u)=2 u f^{2} Q,
\end{array}
$$

and the Fourier series

$$
\begin{array}{r}
f(u)=\sum_{m=1}^{M} f_{G} \sin G u, \quad G=\frac{\pi(2 m-1)}{2 R}, \\
A(u)=\sum_{n=1}^{N} a_{K} \sin K u+\frac{u^{2}}{2} B, \quad K=\frac{\pi n}{R}, \\
B(u)=\sum_{n=1}^{N} a_{K} \frac{2 \sin K u+K u \cos K u}{2 u^{2}}+B, \\
Q(u)=\sum_{n=1}^{N} a_{K} \sin K u-\frac{1-u^{4} / R^{2}}{\kappa u^{2}}, \\
j(u)=\sum_{n=1}^{N} a_{K} \frac{K u \cos K u-\left(4+K^{2} u^{2}\right) \sin K u}{4 u^{4}} .
\end{array}
$$

The equations for the new Fourier coefficients are

$$
\begin{array}{r}
f_{G}=\frac{1}{G^{2}+4 \kappa^{2}}\left[4 \kappa^{2} f_{G}+\frac{2}{N_{r}} \sum_{i=1}^{N_{r}} \sin G u_{i} \times\right. \\
\left.\left(4 u_{i}^{2} \kappa^{2} f\left(1-f^{2}-Q^{2}\right)+f^{\prime} / u_{i}\right)\right], \\
a_{K}=\frac{1}{K^{2}+1}\left[a_{K}+\frac{2}{N_{r}} \sum_{i=1}^{N_{r}} \sin K u_{i} \times\right. \\
\left.\left(\sum_{n^{\prime}=1}^{N} a_{K^{\prime}} \frac{K u_{i} \cos K u_{i}-4 \sin K u_{i}}{u_{i}^{2}}-4 u^{2} Q f^{2}\right)\right] .
\end{array}
$$

For better convergence a term $-4 \kappa f_{G}$ was added on both sides of Eq. (A17) to yield (A24). The corresponding iteration scheme using (A14), (A15) needs a smaller weight $c$ and more iteration steps, but for large $\kappa^{2} / b$ it is faster than the first scheme since it needs less grid points $N_{r}$ to reach the same accuracy.

\section{APPENDIX B: PERIODIC VORTEX LATTICE}

The properties of the ideally periodic FLL within GL theory may be calculated by minimizing the GL free energy of the superconductor, Eq. (2), with respect to appropriate periodic trial functions, e.g., Fourier series with a large number of terms. For the smooth function $\omega=f^{2}(\mathbf{r})$ we write the ansatz

$$
\omega(\mathbf{r})=f^{2}=\sum_{\mathbf{K}} a_{\mathbf{K}}(1-\cos \mathbf{K r} \mathbf{r})
$$

with $\mathbf{r}=(x, y), \mathbf{K}=\left(K_{x}, K_{y}\right)$. In all sums here and below the term $\mathbf{K}=0$ is excluded. For vortex positions $\mathbf{R}=$
$\mathbf{R}_{m n}=\left(m x_{1}+n x_{2}, n y_{2}\right)$ the reciprocal lattice vectors are $\mathbf{K}=\mathbf{K}_{m n}=(2 \pi / S)\left(m y_{2}, n x_{1}+m x_{2}\right)$ with $S=x_{1} y_{2}=$ $\Phi_{0} / B$ the unit cell area and $m, n=0, \pm 1, \pm 2, \ldots$. For the triangular lattice one has $x_{2}=x_{1} / 2, y_{2}=x_{1} \sqrt{3} / 2$, and for the square lattice $x_{2}=0, y_{2}=x_{1}$. For supervelocity $\mathbf{Q}$ and induction $\mathbf{B}=\nabla \times \mathbf{Q}=B(\mathbf{r}) \hat{\mathbf{z}}$ we choose

$$
\begin{aligned}
& B(\mathbf{r})=B+\sum_{\mathbf{K}} b_{\mathbf{K}} \cos \mathbf{K r}, \\
& \mathbf{Q}(\mathbf{r})=\mathbf{Q}_{A}(\mathbf{r})+\sum_{\mathbf{K}} b_{\mathbf{K}} \frac{\hat{\mathbf{z}} \times \mathbf{K}}{K^{2}} \sin \mathbf{K r} .
\end{aligned}
$$

Here $\mathbf{Q}_{A}(x, y)$ is the supervelocity of the Abrikosov $B_{c 2}$ solution, which satisfies

$$
\nabla \times \mathbf{Q}_{A}=\left[B-\Phi_{0} \sum_{\mathbf{R}} \delta_{2}(\mathbf{r}-\mathbf{R})\right] \hat{\mathbf{z}}
$$

where $\delta_{2}(\mathbf{r})=\delta(x) \delta(y)$ is the $2 \mathrm{D}$ delta function. This relation shows that $\mathbf{Q}_{A}$ is the velocity field of a lattice of ideal vortex lines but with zero average rotation. Close to each vortex center one has $\mathbf{Q}_{A}(\mathbf{r}) \approx \hat{\mathbf{z}} \times \mathbf{r}^{\prime} /\left(2 \kappa r^{\prime 2}\right)$ and $\omega(\mathbf{r}) \propto r^{\prime 2}$ with $\mathbf{r}^{\prime}=\mathbf{r}-\mathbf{R}$. In principle $\mathbf{Q}_{A}(\mathbf{r})$ may be expressed as a slowly converging Fourier series by integrating (B4) using $\operatorname{div} \mathbf{Q}=\operatorname{div} \mathbf{Q}_{A}=0$ as in Ref. 15. But it is more convenient to take $\mathbf{Q}_{A}$ from the exact relation

$$
\mathbf{Q}_{A}(\mathbf{r})=\frac{\nabla \omega_{A} \times \hat{\mathbf{z}}}{2 \kappa \omega_{A}}
$$

where $\omega_{A}(x, y)$ is the Abrikosov $B_{c 2}$ solution given by the rapidly converging series (B1) with coefficients 30.31

$$
a_{\mathbf{K}}^{A}=-(-1)^{m+m n+n} \exp \left[-K_{m n}^{2} S /(8 \pi)\right]
$$

for general lattice symmetry, and $a_{\mathbf{K}}^{A}=$ $-(-1)^{\nu^{2}} \exp \left(-\pi \nu^{2} / \sqrt{3}\right)\left(\nu^{2}=m^{2}+m n+n^{2}\right)$ for the triangular lattice. This $\omega_{A}$ is normalized to $\left\langle\omega_{A}(x, y)\right\rangle=1$, which means that $\sum_{\mathbf{K}}^{\prime} a_{\mathbf{K}}^{A}=1$ for any lattice symmetry. Another strange property of the Abrikosov solution (B6) is that $\left(\nabla \omega_{A} / \omega_{A}\right)^{2}-\nabla^{2} \omega_{A} / \omega_{A}=4 \pi / S=$ const, although both terms diverge at the vortex positions; this relation follows from (B4) and (B5) using $B=\Phi_{0} / S=2 \pi /(\kappa S)$. The useful formula (B5) may be proven via the complex $B_{c 2}$ solution $\psi_{A}(x, y)$; it means that near $B_{c 2}$ the third and fourth term in $F$, Eq. (2), are identical.

Approximate solutions $\omega(\mathbf{r})$ and $B(\mathbf{r})$ may be computed by using a finite number of Fourier coefficients $a_{\mathbf{K}}$ and $b_{\mathbf{K}}$ and minimizing the free energy $F\left(B, \kappa, a_{\mathbf{K}}, b_{\mathbf{K}}\right)$ with respect to these coefficients $\frac{15}{}$. However, a much faster and more accurate solution method 17 is to iterate the two GL equations $\delta F / \delta \omega=0$ and $\delta F / \delta \mathbf{Q}=$ 0 written in appropriate form. Namely, the iteration is stable and converges rapidly if one isolates a term $\left(-\nabla^{2}+\right.$ const $)(\omega, \mathbf{Q})$ on the l.h.s. and puts the remaining terms to the r.h.s. as a kind of "inhomogeneity" of such London-like equations, e.g.,

$$
\begin{aligned}
\left(-\nabla^{2}+2 \kappa^{2}\right) \omega & =2 \kappa^{2}\left(2 \omega-\omega^{2}-\omega Q^{2}-g\right) \\
\left(-\nabla^{2}+\bar{\omega}\right) \mathbf{Q}_{b} & =-\omega \mathbf{Q}_{A}-(\omega-\bar{\omega}) \mathbf{Q}_{b}
\end{aligned}
$$


with the abbreviations $g(\mathbf{r})=(\nabla \omega)^{2} /\left(4 \kappa^{2} \omega\right), \mathbf{Q}_{b}=$ $\mathbf{Q}-\mathbf{Q}_{A}, \nabla \times \mathbf{Q}_{b}=B(\mathbf{r})-B$, and $\bar{\omega}=\langle\omega\rangle=$ $\sum_{\mathbf{K}}^{\prime} a_{\mathbf{K}}$. Equations (B7), (B8) introduced some "penetration depths" $\left(2 \kappa^{2}\right)^{-1 / 2}=\xi / \sqrt{2}$ and $\bar{\omega}^{-1 / 2}=\lambda / \bar{\omega}^{1 / 2}$ (in real units), which stabilize the convergence of the iteration. Acting on the Fourier series $\omega$ (B1) and $\mathbf{Q}_{b}$ (B3) the Laplacian $\nabla^{2}$ yields a factor $-K^{2}$; this facilitates the inversion of (B7) and (B8). Using the orthonormality

$$
2\left\langle\cos \mathbf{K r} \cos \mathbf{K}^{\prime} \mathbf{r}\right\rangle=\delta_{\mathbf{K K}^{\prime}}
$$

(for $\mathbf{K} \neq 0$ ) one obtains from (B1), (B2) $a_{\mathbf{K}}=$ $-2\langle\omega(\mathbf{r}) \cos \mathbf{K r}\rangle$ and $b_{\mathbf{K}}=2\langle B(\mathbf{r}) \cos \mathbf{K r}\rangle$. The convergence of the iteration is considerably improved by adding a third equation which minimizes $F$, Eq. (2), with respect to the amplitude of $\omega$, i.e., $\partial F / \partial \bar{\omega}=0$. This step gives the largest decrease of $F$. The resulting three iteration equations for the parameters $a_{\mathbf{K}}$ and $b_{\mathbf{K}}$ then read ${ }^{17}$

$$
\begin{aligned}
& a_{\mathbf{K}}:=\frac{4 \kappa^{2}\left\langle\left(\omega^{2}+\omega Q^{2}-2 \omega+g\right) \cos \mathbf{K r}\right\rangle}{K^{2}+2 \kappa^{2}}, \\
& a_{\mathbf{K}}:=a_{\mathbf{K}} \cdot\left\langle\omega-\omega Q^{2}-g\right\rangle /\left\langle\omega^{2}\right\rangle \\
& b_{\mathbf{K}}:=\frac{-2\langle[(\omega-\bar{\omega}) B(\mathbf{r})+p] \cos \mathbf{K r}\rangle}{K^{2}+\bar{\omega}}
\end{aligned}
$$

with $p=(\nabla \omega \times \mathbf{Q}) \hat{\mathbf{z}}=Q_{x} \partial \omega / \partial y-Q_{y} \partial \omega / \partial x$ and $g=$ $(\nabla \omega)^{2} /\left(4 \kappa^{2} \omega\right)=(\nabla f)^{2} / \kappa^{2}$ as above.

The solutions $\omega(\mathbf{r}), \mathbf{B}(\mathbf{r})$, and $\mathbf{Q}(\mathbf{r})$ are obtained by starting, e.g., with $a_{\mathbf{K}}=(1-b) a_{\mathbf{K}}^{A}$ and $b_{\mathbf{K}}=0$ and then iterating the three equations (B10), (B11), (B12) by turns until the coefficients do not change any more. After typically 25 such triple steps, the solution stays constant to all 15 digits and the GL equations are exactly satisfied. Since all terms in (B10) - (B12) are smooth periodic functions of $\mathbf{r}$, high accuracy is achieved by using a regular spatial $2 \mathrm{D}$ grid, e.g., $x_{i}=(i-1 / 2) x_{1} / N_{x}$ $\left(i=1 \ldots N_{x}\right)$ and $y_{j}=(j-1 / 2) y_{2} /\left(2 N_{y}\right)\left(j=1 \ldots N_{y}\right.$, $\left.2 N_{y} \approx N_{x} y_{2} / x_{1}\right)$ with constant weights $x_{1} / N_{x}$ and $y_{2} /\left(2 N_{y}\right)$. These $N=N_{x} N_{y}=100$ to 5000 grid points fill the rectangular basic area $0 \leq x \leq x_{1}, 0 \leq y \leq y_{2} / 2$, which is valid for any unit cell with the shape of a parallelogram. Spatial averaging $\langle\ldots\rangle$ then just means summing $N$ terms and dividing by $N$.

Best accuracy is achieved by considering all $\mathbf{K}_{m n}$ vectors within a half circle $\left|\mathbf{K}_{m n}\right| \leq K_{\max }$, with $K_{\max }^{2} \approx 20 N / S$ chosen such that the number of the $\mathbf{K}_{m n}$ is slightly less than the number $N$ of grid points. The high precision of this method may be checked with the identity $B(x, y) / B_{c 2}=1-\omega(x, y)$, which is valid at $\kappa=1 / \sqrt{2}$ for all $b$. This relation is confirmed with an error $<10^{-9}$. The equilibrium field $H$ or reversible magnetization $M=B-H$ is computed from Doria's virial theorem, Eq. (3).

\section{APPENDIX C: LONDON THEORY}

The modified London equation for a lattice of straight vortex lines at regular positions $\mathbf{R}=\mathbf{R}_{m n}$ (App. B) is

$$
\left(1-\lambda^{2} \nabla^{2}\right) B(x, y)=\Phi_{0} \sum_{\mathbf{R}} \delta\left(\mathbf{r}-\mathbf{R}_{m n}\right),
$$

where $\delta(x, y)$ is the $2 \mathrm{D}$ delta function. The solution for the magnetic field of one isolated vortex at $\mathbf{R}=0$ is

$$
B_{v}(r)=\left(\Phi_{0} / 2 \pi \lambda^{2}\right) K_{0}(r / \lambda) .
$$

The modified Bessel function

$$
K_{0}(r / \lambda)=\int \frac{d^{2} k}{2 \pi} \frac{\cos \mathbf{k r}}{\lambda^{-2}+k^{2}}
$$

has the derivative $K_{0}(x)^{\prime}=-K_{1}(x)$ with the limits $K_{0}(x \ll 1) \approx-\ln x, K_{1}(x \ll 1) \approx 1 / x$, and for $x \gg 1: 32$

$$
\begin{aligned}
& K_{0}(x) \approx \sqrt{\frac{\pi}{2 x}} e^{-x}\left(1-\frac{1}{8 x}+\frac{9}{128 x^{2}}-\frac{225}{3972 x^{3}}\right), \\
& K_{1}(x) \approx \sqrt{\frac{\pi}{2 x}} e^{-x}\left(1+\frac{3}{8 x}-\frac{15}{128 x^{2}}+\frac{315}{3972 x^{3}}\right) .
\end{aligned}
$$

For a periodic FLL one obtains the Fourier series $B(x, y)$, Eq. (16), which may also be written as a sum over isolated vortex fields, $B(x, y)=\sum_{\mathbf{R}} B_{v}(\mathbf{r}-\mathbf{R})$. Similarly, the free energy of the FLL may be written as a sum of vortex self energies $\left(\Phi_{0} H_{c 1}\right.$ per unit length) plus a double sum over all interactions between two vortices. The average energy density $F$, Eq.(17), then reads

$$
F=B H_{c 1}+\frac{B \Phi_{0}}{4 \pi \lambda^{2} \mu_{0}} \sum_{\mathbf{R}} K_{0}(R / \lambda) .
$$

For the triangular vortex lattice we write $R / \lambda=\nu c$ with $c=a / \lambda=(4 \pi / \sqrt{3})^{1 / 2}\left(b \kappa^{2}\right)^{-1 / 2}(a=$ vortex spacing $)$ and $\nu^{2}=m^{2}+m n+n^{2}=1,3,4,7,7,9, \ldots$ Taking the derivative $H=\partial F / \partial B$ one obtains for $h=H / H_{c 2}$ with $h_{c 1}=H_{c 1} / H_{c 2}$ :

$$
h=h_{c 1}+\frac{3}{\kappa^{2}} \sum_{\nu}\left[K_{0}(\nu c)+\frac{\nu c}{2} K_{1}(\nu c)\right] .
$$

Here the sum is over $\nu=1, \sqrt{3}, 2, \ldots$, i.e. the number of six flux lines per shell is already accounted for. Equation (C6) is still exact. It works for $b \ll 1$ (i.e. for nonoverlapping vortex cores) and for $\kappa>1.4$ (i.e. when the longrange interaction of vortices is purely magnetic $\left.{ }^{11.33}\right)$. With the expansions (C4) one obtains for $x=\nu c \gg 1$ :

$$
h \approx h_{c 1}+\frac{3 \sqrt{\pi}}{2 \kappa^{2}} \sum_{\nu} e^{-x} \sqrt{x}\left[1+\frac{19}{8 x}-\frac{47}{128 x^{2}}\right] .
$$

At very small $b$, namely for $c=a / \lambda \gg 1$, the sum may be restricted to the nearest neighbor shell, i.e. to the first term, $\nu=1$, yielding

$$
h \approx h_{c 1}+\frac{3 \sqrt{\pi c}}{2 \kappa^{2}} e^{-c}\left[1+\frac{19}{8 c}-\frac{47}{128 c^{2}}\right] .
$$


1 A. A. Abrikosov, Zh. Eksp. Teor. Fiz. 32, 1442 (1957) [Sov. Phys. JETP 5, 1174 (1957)].

${ }^{2}$ V. L. Ginzburg and L. D. Landau, Zh. Eksp. Teor. Fiz. 32, 1064 (1950) [English translation in Men of Physics, L. D. Landau, edited by D. ter Haar (New York, Pergamon, 1965), vol. 1, p. 138)].

3 P. G. DeGennes, Superconductivity of Metals and Alloys (New York, Benjamin, 1966).

4 D. Saint-James, G. Sarma, and E. J. Thomas, Type-II Superconductivity (New-York, Benjamin,1969).

5 M. Tinkham, Introduction to Superconductivity (McGraw-Hill: New York 1975).

6 A. L. Fetter and P. C. Hohenberg, in Superconductivity, edited by R. D. Parks (Marcel Dekker, New York, 1969), p. 817.

7 Z. Hao, J. R. Clem, M. W. McElfresh, L. Civale, A. P. Malozemoff, and F. Holtzberg, Phys. Rev. B 43, 2844 (1991).

8 R. A. Klemm and J. R. Clem, Phys. Rev. B 21, 1868 (1980).

9 V. G. Kogan and J. R. Clem, Phys. Rev. B 24, 2497 (1981).

10 R. A. Klemm, Phys. Rev. B 38, 6641 (1988).

11 E. H. Brandt, Rep. Prog. Phys. 58, 1465 (1995). In Eqs. (1.4), (1.7), and (1.8) of that paper the wrong denominator $\left(2 \kappa^{2}-1\right) \beta_{A}$ has to be replaced by $1+\left(2 \kappa^{2}-1\right) \beta_{A}=$ $2 \beta_{A}\left(\kappa^{2}-0.069\right)$.

12 E. H. Brandt, Phys. Rev. B 64, 024505 (2001).

13 D. Ihle, phys. stat. sol. (b) 47, 423 (1971).

14 W. V. Pogosov, K. I. Kugel, A. L. Rakhmanov, and E. H. Brandt, Phys. Rev. B 64, 064517 (2001).

15 E. H. Brandt, phys. stat. sol. (b) 51, 345 (1972).

16 E. H. Brandt, phys. stat. sol. (b) 77, 551 (1976); J. Low
Temp. Phys. 24, 409 and 427 (1976).

17 E. H. Brandt, Phys. Rev. Lett. 78, 2208 (1997).

18 L. Kramer and W. Pesch, Z. Phys. 269, 59 (1974); R. Watts-Tobin, L. Kramer, and W. Pesch, J. Low Temp. Phys. 17, 71 (1974).

19 U. Klein, J. Low Temp. Phys. 69, 1 (1987).

20 M. Ichioka, N. Hayashi, and K. Machida, Phys. Rev. B 55, 6565 (1997)

21 M. Ichioka, N. Hasegawa, and K. Machida, Phys. Rev. B 59, 8902 (1999).

${ }^{22}$ M. Ichioka, N. Enomoto, and K. Machida, J. Phys. Soc. Jpn. 66, 3928 (1997).

23 M. Ichioka and K. Machida, Phys. Rev. B 65, 224517 (2002)

24 P. Lipavský, J. Koláček, K. Morawetz, and E. H. Brandt, Phys. Rev. B 65, 144511 (2002).

25 M. M. Doria, J. E. Gubernatis, and D. Rainer, Phys. Rev. B 39, 9573 (1989); ibd. 41, 6335 (1991); U. Klein and B. Pöttinger, Phys. Rev. B 44, 7704 (1991).

26 E. H. Brandt and A. Seeger, Adv. in Physics 35, 189 (1986)

27 E. H. Brandt, Phys. Rev. B 37, 2349 (1988).

28 J. R. Clem, J. Low Temp. Phys. 18, 424 (1975).

29 A. Yaouanc, P. Dalmas de Réotier, and E. H. Brandt, Phys. Rev. B 55, 11107 (1997).

30 E. H. Brandt, phys. stat. sol. 36, 381, 393 (1969).

31 J. M. Delrieu, J. Low Temp. Phys. 6, 197 (1972).

32 M. Abramowitz and I. A. Stegun, Handbook of Mathematical Functions (Dover, New York, 1968) p. 378.

33 L. Kramer, Phys. Rev. B 11, 3821(1971). 